\title{
Pre-exposure to Fluorouracil Increased Trifluridine Incorporation and Enhanced its Anti-tumor Effect for Colorectal Cancer
}

\author{
TAISUKE BABA, TOSHIO KOKURYO, JUNPEI YAMAGUCHI, YUKIHIRO YOKOYAMA, \\ KEISUKE UEHARA, TOMOKI EBATA and MASATO NAGINO \\ Division of Surgical Oncology, Department of Surgery, \\ Nagoya University Graduate School of Medicine, Nagoya, Japan
}

\begin{abstract}
Background/Aim: Trifluridine/tipiracil (FTD/TPI) is used for metastatic colorectal cancer, that is refractory to 5-fluorouracil (5-FU)-based therapies. However, the impact of pre-exposure to 5-FU on the anti-cancer effect of FTD, which is a key component of FTD/TPI, is unclear. Materials and Methods: The incorporation into DNA and anti-cancer activity of FTD were analyzed in several cancer cell lines under response to FTD treatment with or without 5-FU preexposure. The volumes of tumors in xenografted nude mice were examined among groups that were either untreated or treated with S-1, FTD/TPI or FTD/TPI with pre-exposure to $S$-1. Results: Pre-exposure to 5-FU significantly increased FTD incorporation into DNA and enhanced its anti-cancer effect for viability and proliferation of cancer cells. In the xenograft nude mouse model, the tumor volumes in the FTD/TPI-treated and S-1-pre-exposed group were lower than those in the FTD/TPI-only-treated group. Although both FTD dose and exposure time in the FTD/TPI-treated and S-1-preexposed mice were smaller than those in the FTD/TPI-onlytreated mice, the incorporated FTD in the tumors in the former group was $86.5 \%$ of that in the latter group. Conclusion: Pre-exposure to 5-FU enhanced the incorporation into DNA and the anti-cancer effect of FTD in the context of colorectal cancer. Our data indicate the potential for a new sequential therapy using $S-1$ and FTD/TPI to improve prognosis of colorectal cancer.
\end{abstract}

Correspondence to: Toshio Kokuryo, Division of Surgical Oncology, Nagoya University Graduate School of Medicine, 65 Tsurumai-cho, Showa-ku, Nagoya 466-8550, Japan. Tel: +81 527442222, Fax: +81 527442230, e-mail: kokuryoh@med.nagoyau.ac.jp

Key Words: Colorectal cancer, fluorouracil, pre-exposure, sequential therapy, trifluridine.
Fluorouracil (5-FU) is the key drug used in the treatment for several cancers, including colorectal cancer. Although several regimens can improve the prognosis of these cancer patients, the median overall survival is from 28.7 to 34.2 months, and the objective response rate is from $57.8 \%$ to $65.1 \%$ (1-4).

Trifluridine/tipiracil (FTD/TPI) is an oral combination drug comprising of trifluridine (FTD), an anti-cancer agent, and thymidine phosphorylase inhibitor (TPI), an inhibitor of FTD catabolism (5). FTD/TPI is used for metastatic colorectal cancer, which is refractory to 5-FU-based therapies. FTD is a thymidine-based nucleoside analogue that is metabolized to triphosphate metabolites and incorporated into nucleic acids. This incorporation leads to activation of chk1 phosphorylation, which results in $\mathrm{G}_{2}$ cell-cycle arrest and cell death (6).

As the multiple pathways of proliferation, invasion, and motility are activated in cancer cells, sequential treatment using different drugs at different times is the most effective strategy in cancer therapy. However, the interaction among different anticancer drugs in sequential treatments has not been clarified. Preexposure to 5-FU is thought to affect the inhibitory effect of FTD in 5-FU-refractory colorectal cancer. However, few studies have focused on these issues. In this study, we investigated the effect of 5-FU pre-exposure on FTD administration.

\section{Materials and Methods}

Chemicals and reagents. FTD was purchased from Sigma-Aldrich (St Louis, MO, USA). FTD/TPI was provided by and S-1 was purchased from Taiho Pharmaceutical Co., Ltd. (Tokyo, Japan). 5-FU was purchased from Kyowa Hakko Kirin Co., Ltd. (Tokyo, Japan).

Cell culture. The human colon cancer cell line DLD-1 and the human pancreatic cancer cell lines KLM-1 and PANC-1 were obtained from the Institute of Development, Aging and Cancer (Sendai, Japan). The cells were cultured in RPMI 1640 medium (Invitrogen Life Technologies, Carlsbad, CA, USA) with $10 \%$ heat-inactivated fetal bovine serum (Equitech-Bio, Inc., Kerrville, TX, USA) at $37^{\circ} \mathrm{C}$ in a humidified atmosphere with $5 \% \mathrm{CO}_{2}$. 
Immunofluorescence staining of incorporated FTD. As FTD can be identified by anti-2'-bromodeoxyuridine (BrdU) antibodies, (7) incorporated FTD was assessed by immunofluorescence staining using ab152095 anti-BrdU antibody (Abcam, Cambridge, MA, USA). To evaluate the efficiency of FTD incorporation, DLD-1 cells were treated with different concentrations of FTD $(0.05,0.1,0.5$ and $1 \mu \mathrm{M})$ for $24 \mathrm{~h}$. The cells were also treated with $0.5 \mu \mathrm{M}$ of FTD for different exposure times $(2,6,12$ and $24 \mathrm{~h})$. DLD-1 and PANC- 1 cells were incubated with 5-FU plus FTD $(100 \mu \mathrm{M} 5$-FU and $0.5 \mu \mathrm{M}$ FTD for $24 \mathrm{~h}$ ) and FTD with 5-FU pre-exposure (pre-exposure to $100 \mu \mathrm{M} 5$-FU for $24 \mathrm{~h}$ and then $0.5 \mu \mathrm{M}$ FTD treatment for $24 \mathrm{~h}$ ) as combination treatments. KLM-1 cells were incubated with 5-FU plus FTD (10 $\mu \mathrm{M}$ 5-FU and $0.5 \mu \mathrm{M}$ FTD for $24 \mathrm{~h}$ ) and FTD with 5-FU pre-exposure (pre-exposure to $10 \mu \mathrm{M} 5$-FU for $24 \mathrm{~h}$ and then $0.5 \mu \mathrm{M}$ FTD treatment for $24 \mathrm{~h}$ ) as the combination treatments.

The cells were fixed in $4 \%$ paraformaldehyde, treated in $2 \mathrm{~N} \mathrm{HCl}$ for $30 \mathrm{~min}$ and stained with an anti-BrdU antibody and DAPI. The relative FTD incorporation rate was assessed by the fluorescence intensities of FTD per cell.

Measurement of FTD incorporated into DNA. DNA was extracted from DLD-1 cells or tumors using the NucleoSpin Tissue Kit (Takara Bio Inc., Tokyo, Japan), and DNA concentrations were determined using the Qubit dsDNA BR assay kit (Life Technologies, Carlsbad, CA, USA). The samples were diluted to $10 \mu \mathrm{g} / \mathrm{ml}$ with distilled water and digested to nucleosides using a previously published method (8). The extracted DNA $(2 \mu \mathrm{g})$ and $300 \mu \mathrm{l}$ reaction mixture consisting of $100 \mathrm{mM}$ Tris- $\mathrm{HCl}$ (pH 7.0), $50 \mathrm{mM} \mathrm{NaCl}, 2.5 \mathrm{mM} \mathrm{CaCl}_{2}, 10 \mathrm{mM}$ $\mathrm{MgCl}_{2}, 1 \mathrm{U}$ of DNase I (Takara Bio Inc., Tokyo, Japan), $40 \mu \mathrm{g}$ of phosphodiesterase I (Sigma-Aldrich, St Louis, MO, USA), and $2 \mathrm{U}$ of alkaline phosphatase (Takara Bio Inc., Tokyo, Japan) were incubated at $37^{\circ} \mathrm{C}$ for $2 \mathrm{~h}$. The solution was mixed with $30 \mu \mathrm{l}$ of $4.2 \mathrm{~N}$ perchloric acid and incubated on ice for $10 \mathrm{~min}$. The mixture was neutralized with $\mathrm{K}_{2} \mathrm{HPO}_{4}(2 \mathrm{M}, 90 \mu \mathrm{l})$ and centrifuged at $15,000 \times g$ and $5^{\circ} \mathrm{C}$ for $30 \mathrm{~min}$. Then, $100 \mu \mathrm{l}$ of the supernatant was mixed with $10 \mu \mathrm{l}$ of water, $50 \mu \mathrm{l}$ of $1 \mathrm{M}$ hydrochloric acid, and $20 \mu \mathrm{l}$ of internal standard working solution. The mixture was extracted with $1 \mathrm{ml}$ of methyl tbutyl ether and centrifuged at $15,000 \times g$ and $5^{\circ} \mathrm{C}$ for $5 \mathrm{~min}$. The supernatant was dried under nitrogen at $40^{\circ} \mathrm{C}$, and the residue was reconstituted with $0.1 \mathrm{ml}$ of $0.1 \%$ acetic acid (A) and acetonitrile (B) $(75: 25, \mathrm{v} / \mathrm{v}$ of $\mathrm{A}: \mathrm{B})$. Aliquots $(5 \mu \mathrm{l})$ of reconstituted samples were analyzed on an API 4000 LC/MS/MS system (AB Sciex, Foster City, CA, USA). Incorporation (pmol) of trifluridine into DNA was represented as the amount of FTD per $\mu \mathrm{g}$ of DNA.

WST-1 cell proliferation assay. DLD-1 cells were seeded at 2,000 cells/well into 96 -well plates. After overnight incubation at $37^{\circ} \mathrm{C}$, the cells were treated with 5 -FU $(4 \mu \mathrm{M}$ for $48 \mathrm{~h})$, FTD $(1.4 \mu \mathrm{M}$ for $48 \mathrm{~h})$ or FTD with 5-FU pre-exposure (pre-exposure to $4 \mu \mathrm{M} 5$-FU for $24 \mathrm{~h}$ and then $1.4 \mu \mathrm{M}$ FTD treatment for $24 \mathrm{~h}$ ). The medium was replaced with fresh medium containing $10 \%$ heat-inactivated fetal bovine serum and incubated for $24 \mathrm{~h}$. Ten microliters of WST-1 solution (Roche, Indianapolis, IN, USA) was added to each well, and the cells were incubated for another $90 \mathrm{~min}$, and then the absorbance of each well was determined at 450 and $630 \mathrm{~nm}$ using a microplate reader.

Cell viability assay. Cell death was assessed using the trypan blue dye exclusion assay. DLD- 1 cells were seeded at a density of $7 \times 10^{4}$ cells per well in 12-well plates and incubated overnight at $37^{\circ} \mathrm{C}$. The cells were treated with 5 -FU ( $2 \mu \mathrm{M}$ for $48 \mathrm{~h})$, FTD $(0.4 \mu \mathrm{M}$ for $48 \mathrm{~h})$, or FTD with 5-FU pre-exposure (pre-exposure to $2 \mu \mathrm{M} 5$-FU for $24 \mathrm{~h}$ and then $0.4 \mu \mathrm{M}$ FTD treatment for $24 \mathrm{~h}$ ). Then, the cells were collected and suspended in $1 \%$ trypan blue solution and viable cells were counted.

Histology. Tumor samples were fixed immediately in $10 \%$ buffered formalin, dehydrated in a graded ethanol series, embedded in paraffin, and then stained with hematoxylin and eosin. Cells were counted in 5 high-power-fields.

Immunohistochemistry. Cells were fixed in 4\% paraformaldehyde and stained using anti-Ki-67 rabbit monoclonal antibodies (clone 30-9, Ventana Medical Systems, Tucson, AZ, USA).

Animal studies. All animal experiments were conducted in accordance with the guidelines of the Institute for Laboratory Animal Research, Nagoya University Graduate School of Medicine. Male BALB/c nude mice (8-week-old and weighing 20-25 g) were purchased from SLC Japan (Shizuoka, Japan). The mice were kept in a temperature- and humidity-controlled environment under a $12 \mathrm{~h}$ light-dark cycle with free access to water and food at all times. DLD- 1 cells $\left(3 \times 10^{6}\right)$ were suspended in $100 \mu \mathrm{l}$ of cell matrix and inoculated into the right femoral area of each mouse. FTD/TPI and S-1 were diluted in $250 \mu \mathrm{l}$ of $0.5 \%$ hydroxypropyl methylcellulose (HPMC) and intragastrically administered using an orogastric tube. The mice were randomly divided into four groups: untreated or treated with FTD/TPI $(150 \mathrm{mg} / \mathrm{kg} / \mathrm{day}$, twice a day and five days a week for two weeks), S-1 (8.3 mg/kg/day, once a day and five days in a week for two weeks) or FTD/TPI with S-1 pre-exposure (S-1 treatment for one week and then FTD/TPI treatment for one week). The mice in the untreated group were administered with HPMC alone as a control. The administration started from the inoculation day, and the mice were sacrificed on the 15 th day thereafter. The anti-tumor effect was assessed by the tumor volume (in $\left.\mathrm{mm}^{3}\right)$, calculated as $\left(\mathrm{L}^{2} \times \mathrm{W}\right) / 2$, where $\mathrm{L}$ is the tumor length (in $\mathrm{mm}$ ), and $\mathrm{W}$ is the tumor width (in $\mathrm{mm}$ ).

Statistical analysis. All data are presented as the means \pm standard error (SE). Differences were tested for significance using Bonferroni's method and Tukey-krramer's method. Differences were considered statistically significant at a value of $p<0.05$.

\section{Results}

FTD incorporation in cancer cells. FTD incorporation was analyzed in DLD-1 cells treated with different concentrations of FTD for $24 \mathrm{~h}$. Immunocytochemistry revealed the fluorescent signal of FTD in the cells treated with $0.05 \mu \mathrm{M}$ FTD. FTD incorporation increased in a dose-dependent manner (Figure 1a). FTD incorporation was also assessed in DLD-1 cells treated with $0.5 \mu \mathrm{M}$ of FTD for $2,6,12$ and $24 \mathrm{~h}$. FTD was identified in the cells after $2 \mathrm{~h}$, and its incorporation increased in a time-dependent manner (Figure 1b).

Effect of pre-exposure to 5-FU on FTD incorporation in cancer cells. FTD incorporation was evaluated in DLD-1, PANC-1 and KLM-1 cells that were untreated or treated with FTD (for $24 \mathrm{~h}$ ), 5-FU plus FTD (simultaneous exposure to 5-FU and FTD for $24 \mathrm{~h}$ ), or FTD with 5-FU pre-exposure (pre-exposure to 5-FU for $24 \mathrm{~h}$ and then FTD 


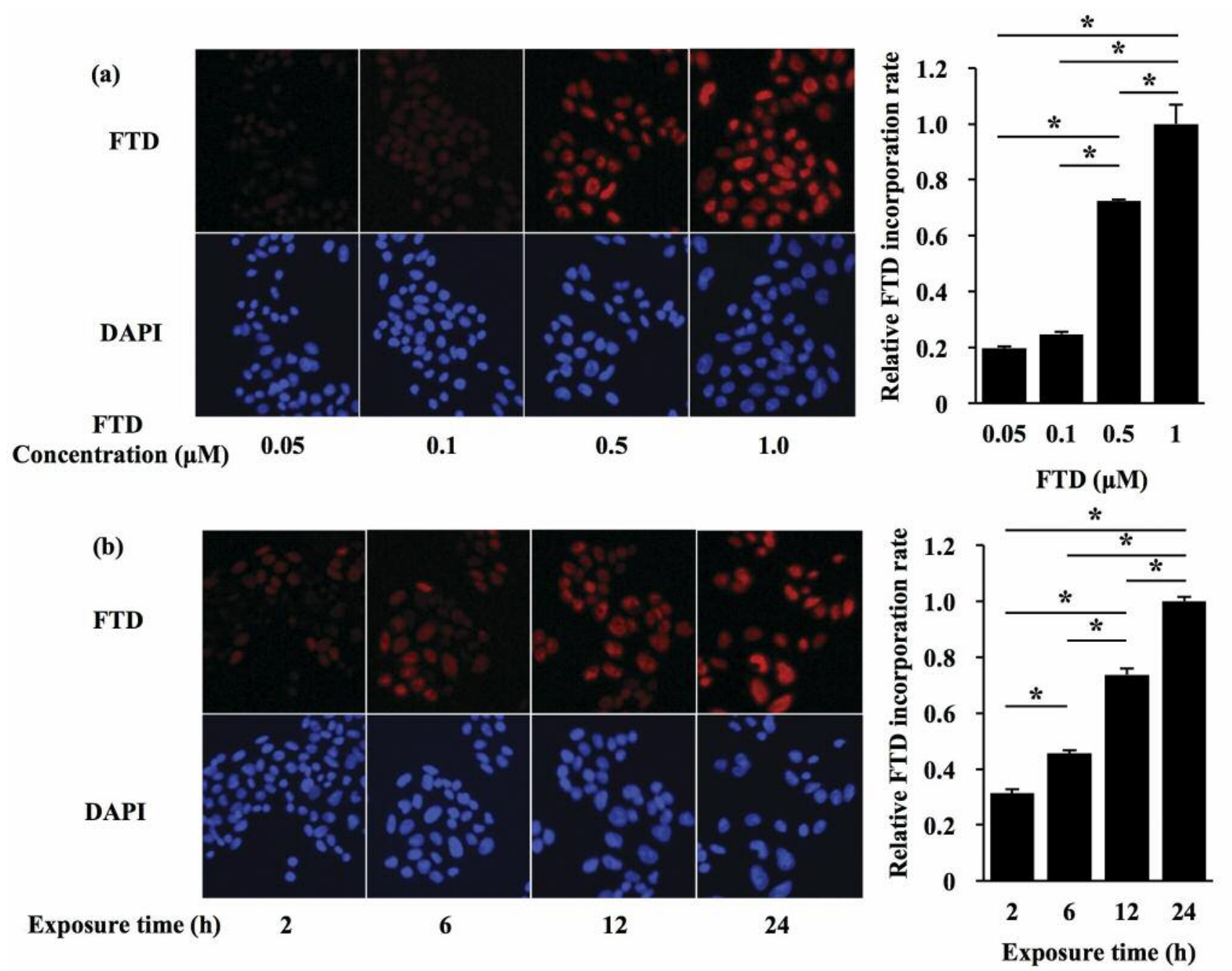

Figure 1. FTD incorporation in cancer cells. (a) Incorporated FTD was detected using ab152095 anti-BrdU antibody. FTD incorporation was assessed in DLD-1 cells that were treated with FTD at 0.05, 0.1, 0.5 and $1 \mu \mathrm{M}$ for 24 h (each group: $n=5$ ). Immunocytochemistry (left); FTD: red, DAPI: blue. Graphs (right) showing the relative FTD incorporation rate in DLD-1 cells. Data are shown relative to the FTD-treated group at $1 \mu M$. ${ }^{*} p<0.01$. (b) FTD incorporation was assessed in DLD-1 cells that were treated with FTD at $0.5 \mu M$ for 2, 6, 12 and 24 h (each group: n=5). Immunocytochemistry (left); FTD: red, DAPI: blue. Graphs (right) showing the relative FTD incorporation rate in DLD-1 cells. Data are shown to relative to the FTD-treated group for $24 h * p<0.01$.

exposure for $24 \mathrm{~h}$ ). As FTD dose and exposure time were the same among the FTD-only, 5-FU plus FTD and FTD with 5-FU pre-exposure groups, we expected that FTD incorporation would be similar among these groups. Unexpectedly, FTD incorporation in the FTD with 5-FU pre-exposure group was significantly higher than that in the FTD-only and 5-FU plus FTD groups (Figure 2a). There were significant differences in the relative intensity of FTD between the groups with FTD treatment with or without preexposure to 5-FU.

FTD incorporation into DNA was analyzed in the untreated or FTD-treated or FTD-treated and 5-FU-pre-exposed DLD-1 cells. Although there was no incorporation of FTD in the untreated group, the incorporation of FTD in the FTD-only and FTD with 5-FU pre-exposure groups were $24.8 \pm 5.0$ and $46.6 \pm 2.7 \mathrm{pmol} / \mu \mathrm{g}$ DNA, respectively (Figure $2 \mathrm{~b}$ ). The average FTD incorporation in the FTD with 5-FU pre-exposure group was 1.9-times higher than that in the FTD-only group, indicating that pre-exposure to 5-FU significantly enhanced FTD incorporation into DNA.

Enhancement of the anti-cancer effect of FTD by pre-exposure to 5-FU. Cell proliferation was assessed in DLD-1 cells that were treated with 5-FU ( $4 \mu \mathrm{M}$ for $48 \mathrm{~h}$ ), FTD (1.4 $\mu \mathrm{M}$ for $48 \mathrm{~h}$ ) or FTD with 5-FU pre-exposure (pre-exposure to $4 \mu \mathrm{M}$ 5-FU for $24 \mathrm{~h}$ and then $1.4 \mu \mathrm{M}$ FTD treatment for $24 \mathrm{~h}$ ). 

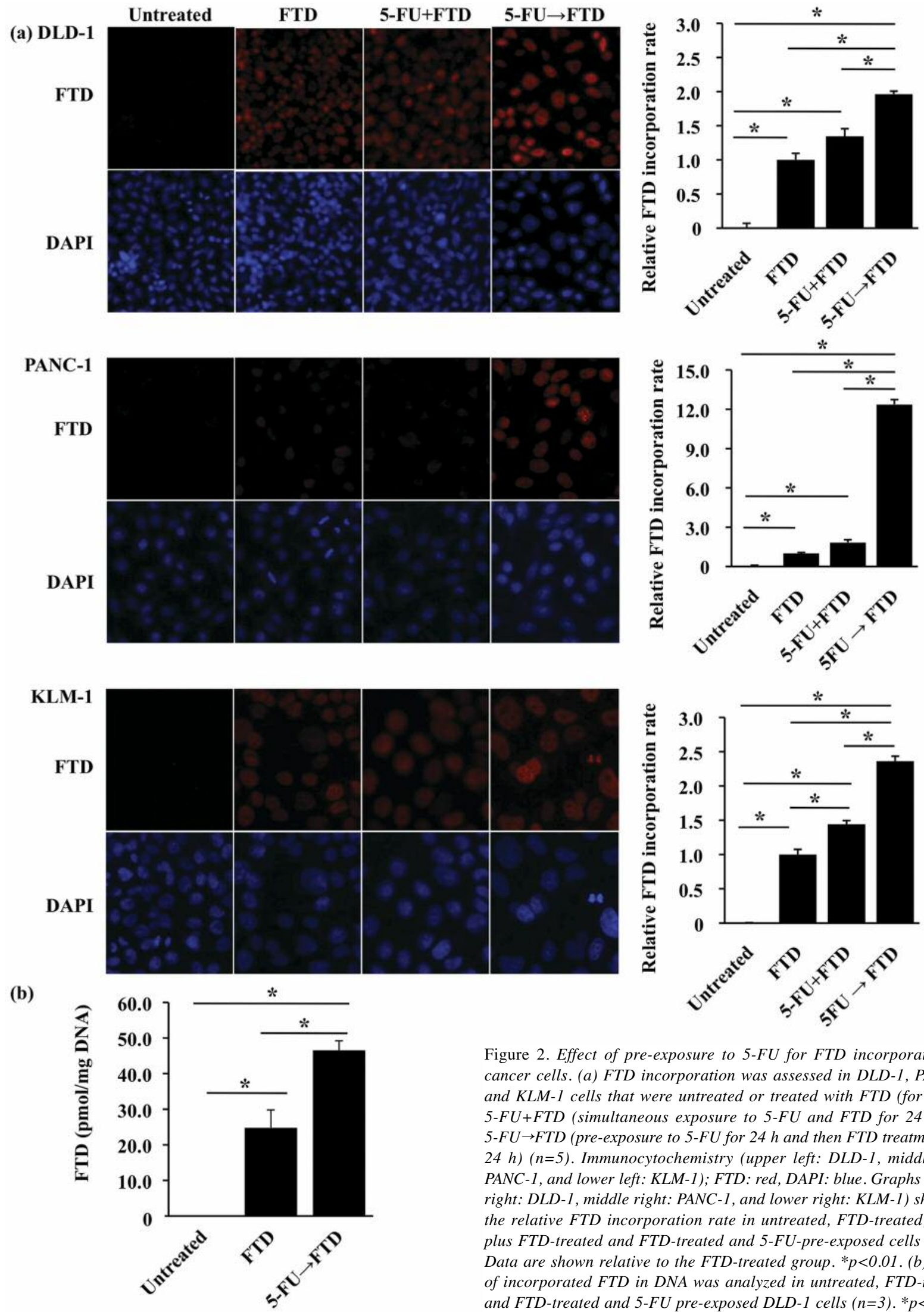

Figure 2. Effect of pre-exposure to 5-FU for FTD incorporation in cancer cells. (a) FTD incorporation was assessed in DLD-1, PANC-1 and KLM-1 cells that were untreated or treated with FTD (for $24 \mathrm{~h}$ ), 5-FU+FTD (simultaneous exposure to 5-FU and FTD for $24 \mathrm{~h}$ ), or 5-FU $\rightarrow F T D$ (pre-exposure to 5-FU for $24 \mathrm{~h}$ and then FTD treatment for $24 h)(n=5)$. Immunocytochemistry (upper left: DLD-1, middle left: PANC-1, and lower left: KLM-1); FTD: red, DAPI: blue. Graphs (upper right: DLD-1, middle right: $P A N C-1$, and lower right: KLM-1) showing the relative FTD incorporation rate in untreated, FTD-treated, 5-FU plus FTD-treated and FTD-treated and 5-FU-pre-exposed cells $(n=5)$. Data are shown relative to the FTD-treated group. ${ }^{*} p<0.01$. (b) Level of incorporated FTD in DNA was analyzed in untreated, FTD-treated and FTD-treated and 5-FU pre-exposed DLD-1 cells $(n=3)$. ${ }^{*} p<0.01$. 
(a)

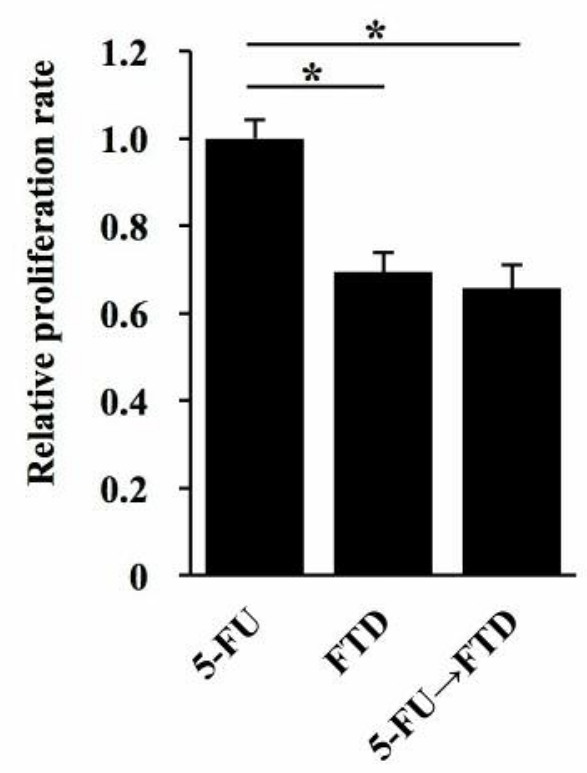

(b)

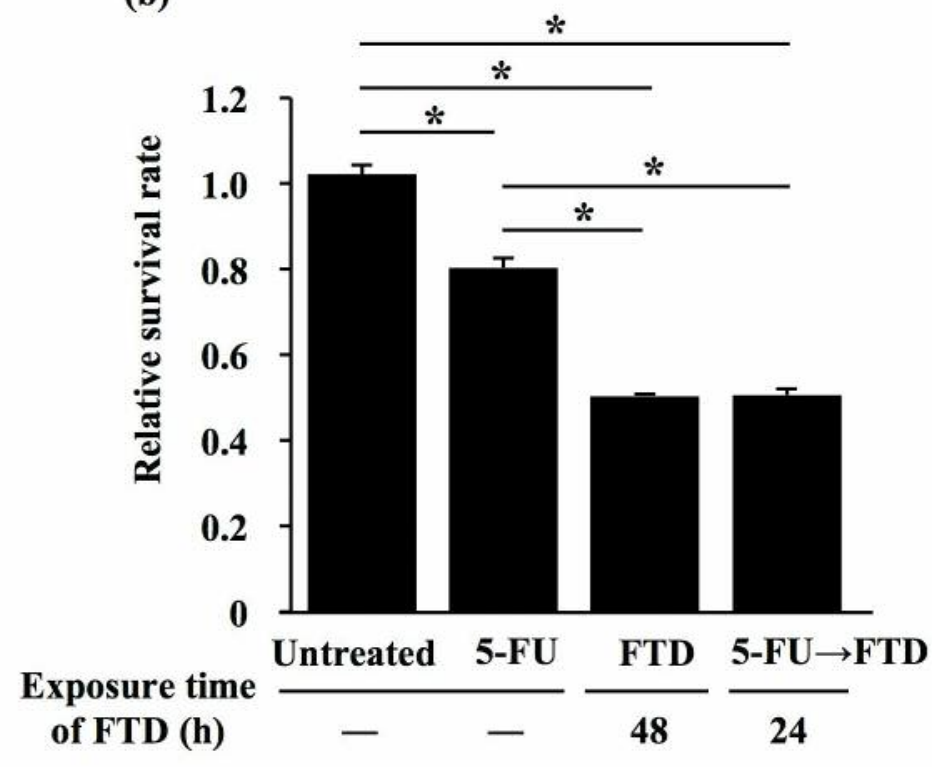

(c)

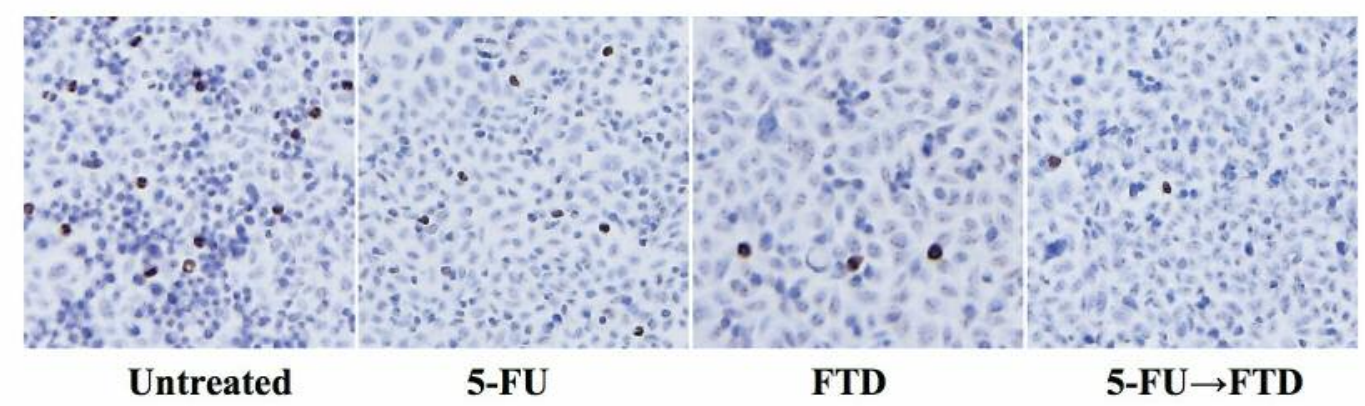

(d)

$*$

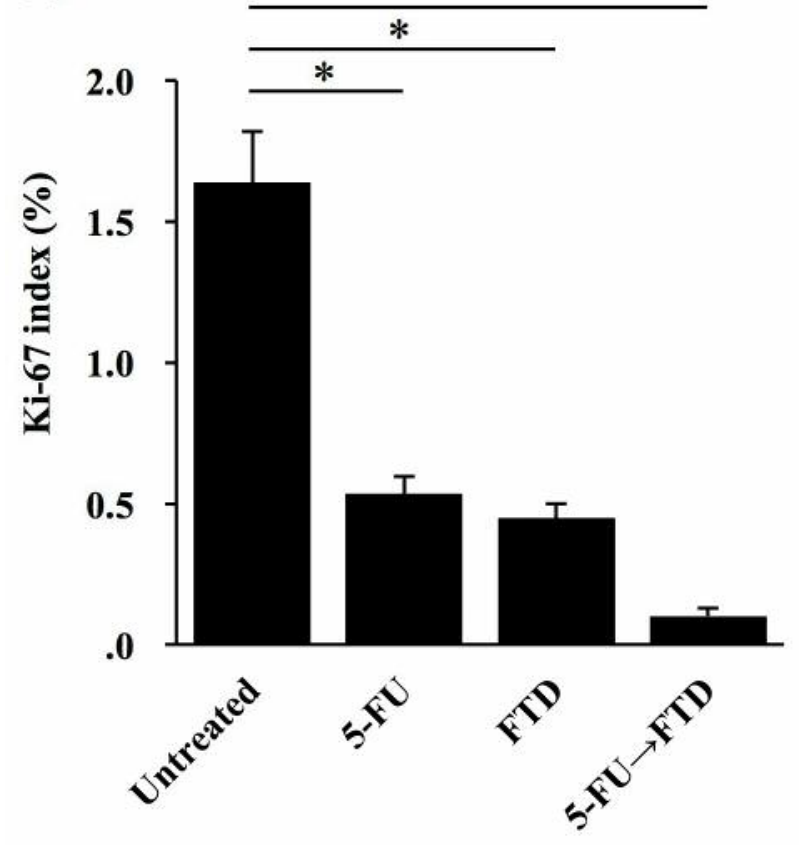

Figure 3. Enhancement of the anti-cancer effect of FTD by pre-exposure to 5-FU. (a) Cell proliferation was analyzed using the WST1 assay in DLD-1 cells that were treated with 5-FU (4 $\mu M$ for $48 \mathrm{~h}), F T D(1.4 \mu \mathrm{M}$ for $48 h)$ or FTD with 5-FU pre-exposure $(5-F U \rightarrow F T D$, pre-exposure to $4 \mu M$ 5-FU for $24 h$ and then $1.4 \mu M$ FTD treatment for $24 h)(n=8)$. Data are shown relative to the $5-F U$ group. ${ }^{*} p<0.01$. (b) Cell viability was analyzed using the trypan blue dye exclusion assay in untreated, 5 $F U$-treated ( $2 \mu M$ for $48 h$ ), FTD-treated $(0.4 \mu M$ for $48 h)$ and FTDtreated and 5-FU-pre-exposed (5-FU $\rightarrow F T D$, pre-exposure to $2 \mu M$ 5$F U$ for $24 h$ and then $0.4 \mu M$ FTD treatment for 24 h) DLD-1 cells $(n=3)$. Data are shown relative to the untreated group. ${ }^{*} p<0.01$. (c) Ki67 expression was stained immunohistochemically in untreated, 5-FUtreated, FTD-treated and FTD-treated and 5-FU-pre-exposed DLD-1 cells. (d) Graphs showing Ki-67-labeling index in untreated, 5-FUtreated, FTD-treated and FTD-treated and 5-FU-pre-exposed DLD-1 cells. ${ }^{*} p<0.01$. 
(a)

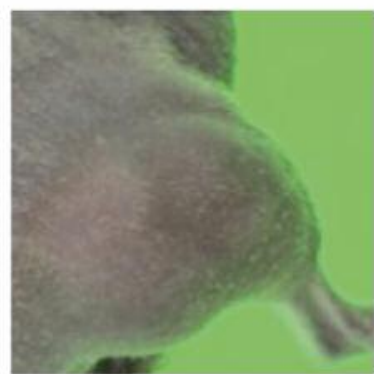

Untreated

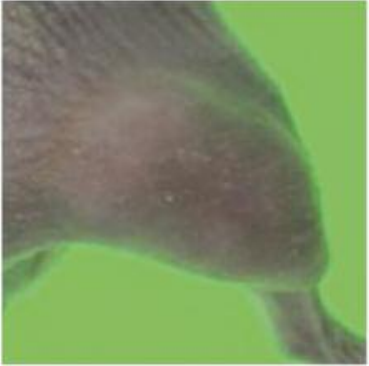

S-1

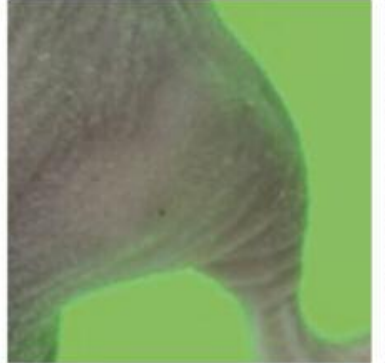

FTD/TPI

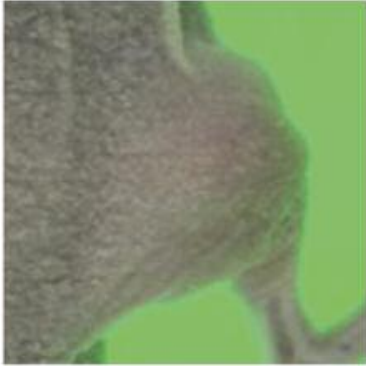

$\mathrm{S}-1 \rightarrow$ FTD/TPI

(b)

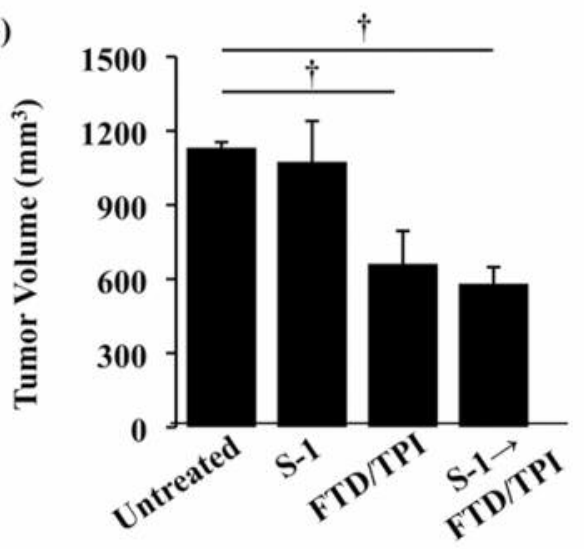

(c)
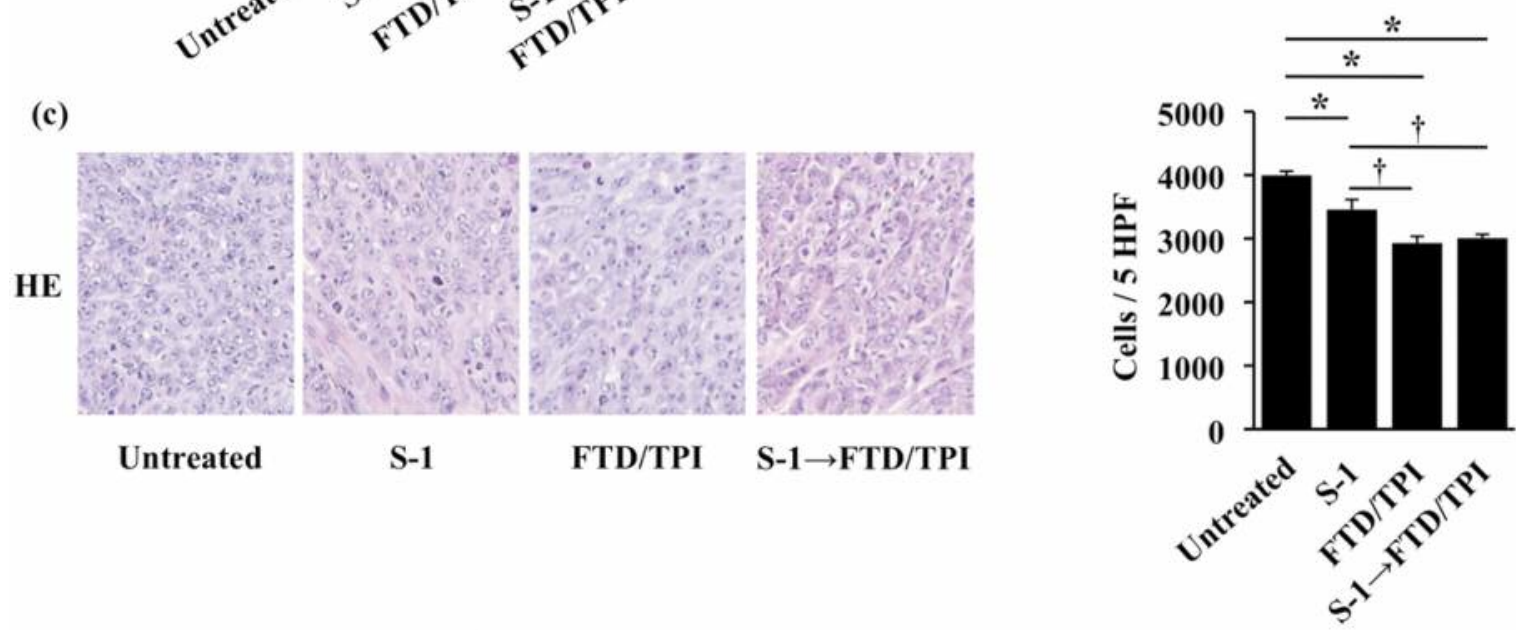

(d)

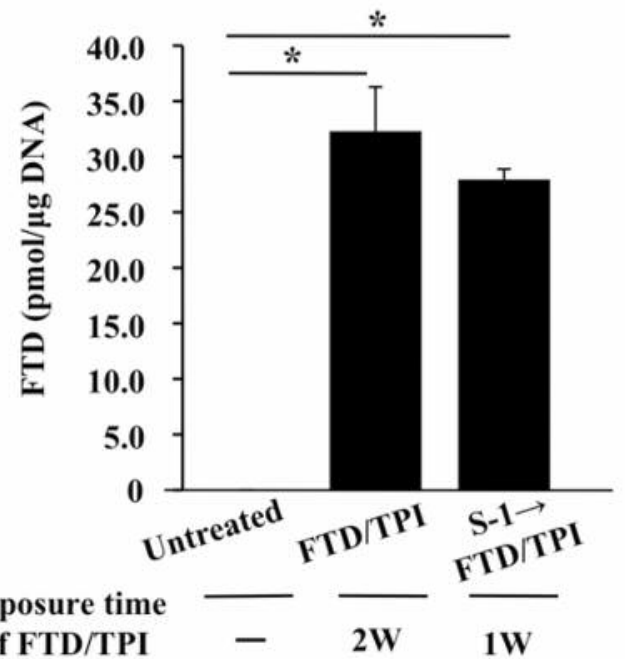

Figure 4. Sequential treatment with $S-1$ and FTD/TPI in a xenograft nude mouse model. (a) Representative images of DLD-1 xenograft tumors that were untreated or treated with FTD/TPI $(150 \mathrm{mg} / \mathrm{kg} /$ day, twice a day and five days a week for two weeks), $S-1$ ( $8.3 \mathrm{mg} / \mathrm{kg} /$ day, once a day and five days a week for two weeks) or FTD/TPI with S-1 pre-exposure $(S-1 \rightarrow F T D / T P I, S-1$ treatment for one week and then FTD/TPI treatment for one week) ( $n=5$ for each treatment). (b) Graphs showing the volume of DLD-1 xenograft tumors that were untreated or treated with FTD/TPI, S-1or FTD/TPI with S-1 pre-exposure. ${ }^{\dagger} p<0.05$. (c) Histology by hematoxylin and eosin staining (left) and cell number (right) of DLD-1 xenograft tumors, that were untreated or treated with $S-1, F T D / T P I$, or FTD/TPI with $S-1$ pre-exposure. ${ }^{\dagger} p<0.05$, ${ }^{*} p<0.01$. (d) Level of incorporated FTD in DNA was analyzed in DLD-1 xenograft tumors, that were untreated or treated with FTD/TPI or FTD/TPI with $S$-1 pre-exposure $(n=5) .{ }^{*} p<0.01$. W: week. 
Interestingly, although the FTD exposure time in the FTD with 5-FU pre-exposure group was shorter than that in the FTD-only group, there was no significant difference in the proliferation rates between the two groups (Figure 3a).

Cell viability was assessed in DLD-1 cells that were untreated or treated with 5-FU ( $2 \mu \mathrm{M}$ for $48 \mathrm{~h})$, FTD $(0.4 \mu \mathrm{M}$ for $48 \mathrm{~h}$ ) or FTD with 5-FU pre-exposure (pre-exposure to $2 \mu \mathrm{M} 5$-FU for $24 \mathrm{~h}$ and then $0.4 \mu \mathrm{M}$ FTD treatment for $24 \mathrm{~h}$ ). Although 5-FU treatment for $48 \mathrm{~h}$ hardly affected the viability of DLD-1 cells, FTD treatment for $48 \mathrm{~h}$ significantly suppressed the viability of these cells. Although the FTD exposure time in the FTD with 5-FU pre-exposure group was half that in the FTD-only group, cell viability was similar between the two groups (Figure 3b).

To evaluate the inhibitory effects of the drugs on cell growth, the Ki-67-labeling index was assessed in DLD-1 cells treated with 5-FU (2 $\mu \mathrm{M}$ for $48 \mathrm{~h})$, FTD $(0.4 \mu \mathrm{M}$ for $48 \mathrm{~h})$, or FTD with 5-FU pre-exposure (pre-exposure to $2 \mu \mathrm{M} 5$-FU for $24 \mathrm{~h}$ and then $0.4 \mu \mathrm{M}$ FTD treatment for $24 \mathrm{~h}$ ). The indices in the untreated, 5-FU, FTD and FTD with 5-FU pre-exposure group were $1.6 \pm 0.18,0.53 \pm 0.06,0.45 \pm 0.05$ and $0.10 \pm 0.03$, respectively (Figure 3c, d). The index was lower in the FTD with 5-FU pre-exposure group than that in the untreated, 5-FUtreated, or FTD-treated group. However, the differences did not reach statistical significance between FTD with 5-FU preexposure group and FTD-only-treated group.

Sequential treatment with $S-1$ and FTD/TPI in a xenograft nude mouse model. $\mathrm{S}-1$ is a prodrug of 5-FU, whereas FTD/TPI is a FTD-based oral drug, and both are commercially available. Therefore, we used these drugs for an in vivo study. DLD-1 cells were inoculated into the right femoral area of nude mice. The mice were randomly assigned to the following four groups: untreated, S-1-treated, FTD/TPI-treated, and FTD/TPI-treated and S-1-pre-exposed groups $(n=5$ in each group). The tumor volumes in the untreated and $\mathrm{S}-1-$, FTD/TPI-treated and FTD/TPI-treated and S-1-pre-exposed groups were $1132 \pm 22,1,075 \pm 165,662 \pm 132$ and $582 \pm 66 \mathrm{~mm}^{3}$, respectively (Figure $4 \mathrm{a}, \mathrm{b}$ ). The tumor volume in the FTD/TPI with S-1 pre-exposure group was lower than that in the FTD/TPI group. However, the differences did not reach statistical significance.

Based on histology, the proportion of cancer cells was decreased in the tumors in the S-1, FTD/TPI and FTD/TPI with $\mathrm{S}-1$ pre-exposure groups compared with that in the untreated group (Figure 4c). However, there were significant differences among the S-1, FTD/TPI and FTD/TPI with S-1 pre-exposure groups.

To further characterize the effect of S-1 (a 5-FU-based oral drug) on FTD incorporation in xenograft tumors, FTD incorporation into tumor DNA was evaluated among the untreated, FTD/TPI-treated and FTD/TPI treated and S-1pre-exposed mice. FTD incorporation in the untreated,
FTD/TPI-treated and FTD/TPI-treated and S-1-pre-exposed mice was $0,32.3 \pm 3.99$ and $28.0 \pm 0.84 \mathrm{pmol} / \mu \mathrm{g}$ DNA, respectively (Figure 4d). The total dose of FTD in the FTD/TPI with S-1 pre-exposure group was half that in the FTD/TPI-only group. However, the incorporation of FTD in the FTD/TPI with S-1 pre-exposure group was $86.5 \%$ of that in the FTD/TPI-only group.

\section{Discussion}

In this study, we demonstrated that pre-exposure to 5-FU increased FTD incorporation into DNA of human colon cancer cells and pancreatic cancer cells. In addition, this pre-exposure enhanced the anti-tumor effect of FTD in a xenograft nude mouse model.

It has been reported that TS inhibitors including 5-FU and 5-fluoro-2'-deoxyuridine modulate the incorporation of thymidine analogs, such as BrdU and 5-iodo-2'-deoxyuridine, $(9,10)$ as FTD is a thymidine-based nucleoside analogue, 5FU is considered to increase its incorporation into DNA.

FTD incorporation into DNA is a key mechanism of its cytotoxicity, (11) and the anti-tumor activity of FTD has been reported to be directly proportional to the amount of FTD incorporated into DNA (8). In our study, although the FTD exposure time in the FTD treatment with 5-FU pre-exposure group was half than that in the FTD-only treatment group, proliferation and viability were strongly inhibited in the FTD treatment with 5-FU pre-exposure group. Accordingly, preexposure to 5-FU improves the anti-cancer effect of FTD.

Interestingly, FTD incorporation into DNA was different between the 5-FU plus FTD and FTD with 5-FU pre-exposure groups. Some researchers have reported that the anti-cancer effect of FTD in combination therapies is dependent on the sequence of FTD administration (12-14). For example, exposure to docetaxel before FTD administration was synergistic, whereas the reverse order was antagonistic in terms of anti-cancer effects (14). Benson et al. reported that depletion of deoxythymidine triphosphate (dTTP) by the inhibition of thymidylate synthetase (TS) enhanced the incorporation of a thymidine analogs into DNA (15). Although the enhancement of the incorporation of thymidine analogs differs between thymidine analogs and TS inhibitors, (10) depletion of dTTP may be associated with the enhancement of FTD incorporation.

Cancer cells become depleted of thymidine when TS is inhibited by 5-FU. Under these conditions, thymidine analogs, such as FTD are rapidly incorporated into cells. However, there is lag between the time of 5-FU administration and the time when thymidine is depleted. In this regard, the simultaneous administration of 5-FU and FTD may not provide enough time for the achievement of thymidine depletion. Therefore, the sequence of drug administration is important for the anti-cancer effect of FTD in combination treatment. 
To further confirm the effect of pre-exposure to 5-FU on FTD incorporation, we performed in vivo experiments using S1 and FTD/TPI. Although both FTD dose and exposure time in the FTD/TPI-treated and S-1-pre-exposed mice were smaller than those in the FTD/TPI-only-treated mice, similar antitumor effects were identified between two groups. These data suggested that sequential therapy using S-1 and FTD/TPI may be a novel strategy to enhance the anti-tumor effect and reduce the dose of each drug for colorectal cancer. However, the most effective dose and exposure time for $\mathrm{S}-1$ to be used before FTD/TPI administration are not known. As FTD/TPI is a combination drug composed of FTD and TPI, the effect of preexposure to S-1 to TPI should also be analyzed. Further investigations are required concerning these issues before the clinical application of the new therapeutic strategy.

In summary, this study demonstrated that pre-exposure to 5-FU enhanced the incorporation and anti-tumor effect of FTD in the context of colorectal cancer. Our data indicate the potential for a new sequential therapy using S-1 and FTD/TPI to improve the prognosis of colorectal cancer.

\section{Conflicts of Interest}

This study was funded by Taiho Pharmaceutical Co. Ltd. (Tokyo, Japan). The sponsor had no role in the study design, study performance, data collection, management and interpretation, or preparation or approval of the article.

\section{Acknowledgements}

The Authors would like to thank the Tokushima Laboratory of Taiho Pharmaceutical, Japan for the excellent technical assistance they offered in the experiments.

\section{References}

1 Yamada Y, Takahari D, Matsumoto H, Baba H, Nakamura M, Yoshida K, Yoshida M, Iwamoto S, Shimada K, Komatsu Y, Sasaki Y, Satoh T, Takahashi K, Mishima H, Muro K, Watanabe M, Sakata Y, Morita S, Shimada Y and Sugihara K: Leucovorin, fluorouracil, and oxaliplatin plus bevacizumab versus $\mathrm{S}-1$ and oxaliplatin plus bevacizumab in patients with metastatic colorectal cancer (SOFT): an open-label, non-inferiority, randomised phase 3 trial. Lancet Oncol 14: 1278-1286, 2013.

2 Cremolini C, Loupakis F, Antoniotti C, Lupi C, Sensi E, Lonardi S, Mezi S, Tomasello G, Ronzoni M, Zaniboni A, Tonini G, Carlomagno C, Allegrini G, Chiara S, D'Amico M, Granetto C, Cazzaniga M, Boni L, Fontanini G and Falcone A: FOLFOXIRI plus bevacizumab versus FOLFIRI plus bevacizumab as first-line treatment of patients with metastatic colorectal cancer: updated overall survival and molecular subgroup analyses of the openlabel, phase 3 TRIBE study. Lancet Oncol 16: 1306-1315, 2015.

3 Schwartzberg LS, Rivera F, Karthaus M, Fasola G, Canon J-L, Hecht JR, Yu H, Oliner KS and Go WY: PEAK: a randomized, multicenter phase II study of panitumumab plus modified fluorouracil, leucovorin, and oxaliplatin (mFOLFOX6) or bevacizumab plus mFOLFOX6 in patients with previously untreated, unresectable, wild-type KRAS exon 2 metastatic colorectal cancer. J Clin Oncol 32: 2240-2247, 2014.
4 Heinemann V, Weikersthal von LF, Decker T, Kiani A, VehlingKaiser U, Al-Batran S-E, Heintges T, Lerchenmüller C, Kahl C, Seipelt G, Kullmann F, Stauch M, Scheithauer W, Hielscher J, Scholz M, Müller S, Link H, Niederle N, Rost A, Höffkes H-G, Moehler M, Lindig RU, Modest DP, Rossius L, Kirchner T, Jung A and Stintzing S: FOLFIRI plus cetuximab versus FOLFIRI plus bevacizumab as first-line treatment for patients with metastatic colorectal cancer (FIRE-3): a randomised, open-label, phase 3 trial. Lancet Oncol 15: 1065-1075, 2014.

5 Lenz H-J, Stintzing S and Loupakis F: TAS-102, a novel antitumor agent: a review of the mechanism of action. Cancer Treat Rev 41: 777-783, 2015.

6 Matsuoka K, Iimori M, Niimi S, Tsukihara H, Watanabe S, Kiyonari S, Kiniwa M, Ando K, Tokunaga E, Saeki H, Oki E, Maehara $\mathrm{Y}$ and Kitao $\mathrm{H}$ : Trifluridine induces p53-dependent sustained $\mathrm{G}_{2}$ phase arrest with its massive misincorporation into DNA and few DNA strand breaks. Mol Cancer Ther 14: 10041013, 2015.

7 Kitao H, Morodomi Y, Niimi S, Kiniwa M, Shigeno K, Matsuoka $\mathrm{K}$, Kataoka Y, Iimori M, Tokunaga E, Saeki H, Oki E and Maehara Y: The antibodies against 5-bromo-2'-deoxyuridine specifically recognize trifluridine incorporated into DNA. Sci Rep 6: 25286, 2016.

8 Tanaka N, Sakamoto K, Okabe H, Fujioka A, Yamamura K, Nakagawa F, Nagase H, Yokogawa T, Oguchi K, Ishida K, Osada A, Kazuno H, Yamada Y and Matsuo K: Repeated oral dosing of TAS-102 confers high trifluridine incorporation into DNA and sustained antitumor activity in mouse models. Oncol Rep 32: 2319-2326, 2014.

9 Stetson PL, Normolle DP, Knol JA, Johnson NJ, Yang ZM, Sakmar E, Prieskorn D, Terrio P, Knutsen CA and Ensminger WD: Biochemical modulation of 5-bromo-2"-deoxyuridine and 5-iodo-2-"deoxyuridine incorporation into DNA in VX2 tumorbearing rabbits. J Natl Cancer Inst 83: 1659-1667, 1991.

10 Sotos GA, Grogan L and Allegra CJ: Preclinical and clinical aspects of biomodulation of 5-fluorouracil. Cancer Treat Rev 20: 11-49, 1994.

11 Peters GJ: Therapeutic potential of TAS-102 in the treatment of gastrointestinal malignancies. Ther Adv Med Oncol 7: 340-356, 2015.

12 Temmink OH, Hoebe EK, Fukushima $M$ and Peters GJ: Irinotecan-induced cytotoxicity to colon cancer cells in vitro is stimulated by pre-incubation with trifluorothymidine. Eur J Cancer 43: 175-183, 2007.

13 Temmink OH, Hoebe EK, van der Born K, Ackland SP, Fukushima $\mathrm{M}$ and Peters GJ: Mechanism of trifluorothymidine potentiation of oxaliplatin-induced cytotoxicity to colorectal cancer cells. Br J Cancer 96: 231-240, 2007.

14 Bijnsdorp IV, Kruyt FA, Gokoel S, Fukushima M and Peters GJ: Synergistic interaction between trifluorothymidine and docetaxel is sequence dependent. Cancer Sci 99: 2302-2308, 2008.

15 Benson $\mathrm{AB}$, Trump DL, Cummings $\mathrm{KB}$ and Fischer $\mathrm{PH}$ : Modulation of 5-iodo-2'-deoxyuridine metabolism and cytotoxicity in human bladder cancer cells by fluoropyrimidines. Biochem Pharmacol 34: 3925-3931, 1985. 